\title{
On the Narumi-Katayama Index of Composite Graphs ${ }^{\dagger}$
}

\author{
Mohammad Ali Hosseinzadeh, ${ }^{a}$ Ali Iranmanesh,,${ }^{\text {a, }}$ \\ and Tomislav Došlićc \\ ${ }^{\mathrm{a}}$ Department of Mathematics, Faculty of Mathematical Sciences, Tarbiat Modares University,
P.O. Box:14115-137, Tehran, Iran
}

RECEIVED JULY 8, 2013; REVISED DECEMBER 5, 2013; ACCEPTED DECEMBER 5, 2013

\begin{abstract}
The Narumi-Katayama index of a graph $G$, denoted by $N K(G)$, is equal to the product of degrees of vertices of $G$. In this paper we investigate its behavior under several binary operations on graphs. We present explicit formulas for its values for composite graphs in terms of its values for operands and some auxiliary invariants. We demonstrate applications of our results to several chemically relevant classes of graphs and show how the Narumi-Katayama index can be used as a measure of graph irregularity. (doi: $10.5562 /$ cca2329)
\end{abstract}

Keywords: Narumi-Katayama index, composite graph, graph product

\section{INTRODUCTION}

Several hundreds of topological invariants of molecular graphs have been defined and employed in the QSAR/QSPR research during the last couple of decades. ${ }^{11}$ One of the simplest, defined as the product of degrees of all vertices, was introduced by Narumi and Katayama in 1984 and named, accordingly, the "simple topological index". ${ }^{10}$ In the subsequent papers the more informative name "Narumi-Katayama index" was introduced and became established, so we use it in the present paper. In the beginning, the index attracted only a moderate attention, ${ }^{4,6}$ but recently a number of papers appeared studying its various mathematical properties (such as the extremal graphs and values) ${ }^{5}$ and its values over special classes of graphs. ${ }^{12}$ Further, it also spawned various generalizations such as the degree product polynomial considered recently by Klein and Rosenfeld. ${ }^{8,9}$ This paper aims to further contribute to the better understanding of the Narumi-Katayama index by investigating its behavior under several binary operations on graphs. We start by defining the terms.

A molecular graph is a simple graph such that its vertices correspond to the atoms and the edges to the bonds. ${ }^{13}$ For a given graph $G$ we denote its vertex set by $V(G)$ and its edge set by $E(G)$. The number of vertices is denoted by $n$. If we consider several graphs, $G_{1}, \ldots, G_{k}$, the quantities pertaining to a given graph are denoted by the corresponding subscript. The degree $d_{G}(v)$ of a vertex $v \in V(G)$ is the number of neighbors of $v$ in $G$. When the graph $G$ is clear from the context, we omit the subscript.

The Narumi-Katayama index of a graph $G$ is defined as the product of degrees of all its vertices,

$$
N K(G)=\prod_{i=1}^{n} d_{G}\left(v_{i}\right)
$$

It is clear from the definition that we can restrict our attention to connected graphs, since for a graph with several connected components its Narumi-Katayama index is equal to the product of the indices of components. (This restriction also takes care of graphs with isolated vertices, among which the index cannot discriminate.)

Let $U=\left\{u_{1}, u_{2}, \ldots, u_{k}\right\}$ be a subset of $V(G)$. We define the truncated Narumi-Katayama index (with respect to $U$ ) as

$$
N K^{(U)}(G)=\prod_{v \in V(G)-U} d_{G}(v)
$$

In the case when $U$ is the empty set, we obtain $N K^{(ø)}(G)=N K(G)$. Note that here the vertices of $\mathrm{U}$ are not deleted from $V(G)$, and the degrees of vertices not in $U$ are not affected. The truncated Narumi-Katayama index will enable us to express some of our results in a more compact form.

\footnotetext{
$\dagger$ Dedicated to Professor Douglas Jay Klein on the occasion of his $70^{\text {th }}$ birthday.

* Author to whom correspondence should be addressed. (E-mail: iranmanesh@modares.ac.ir)
} 


\section{COMPOSITE GRAPHS}

Many interesting classes of graphs arise from simpler graphs via binary operations sometimes known as graph products. (We refer the reader to a monograph by Imrich and Klavžar ${ }^{7}$ for a comprehensive introduction.) Our aim here is to study how the Narumi-Katayama indices of such graphs can be expressed in terms of NarumiKatayama indices of operands and some auxiliary invariants. We start by three simple operations on the union of two graphs.

\section{Splice, Link and Gate}

Let $G_{1}$ and $G_{2}$ are two graphs with disjoint vertex sets. For given vertices $v_{1} \in V\left(G_{1}\right)$ and $v_{2} \in V\left(G_{2}\right)$ the splice of $G_{1}$ and $G_{2}$ by vertices $v_{1}$ and $v_{2},\left(G_{1} \cdot G_{2}\right)\left(v_{1}, v_{2}\right)$, is defined by identifying the vertices $v_{1}$ and $v_{2}$ in the union of $G_{1}$ and $G_{2}$. Similarly, the link of $G_{1}$ and $G_{2}$ by vertices $v_{1}$ and $v_{2}$ is defined as the graph $\left(G_{1} \sim G_{2}\right)\left(v_{1}, v_{2}\right)$ obtained by joining $v_{1}$ and $v_{2}$ by an edge in the union of these graphs. We shorten the notation to $G_{1} \cdot G_{2}$ and $G_{1} \sim G_{2}$ when the vertices $v_{1}, v_{2}$ are clear from the context. (These two operations also appear in the literature under different names; we follow here the terminology introduced in Ref. 2.)

The gate $\left(G_{1} \| G_{2}\right)\left(u_{1}, v_{1} ; u_{2}, v_{2}\right)$ is obtained from $G_{1}$ and $G_{2}$ by identifying the edges $u_{1} v_{1}$ of $G_{1}$ and $u_{2} v_{2}$ of $G_{2}$ so that $u_{1}$ is identified with $u_{2}$ and $v_{1}$ with $v_{2}$. We denote the end-vertices of the identified edge in $G_{1} \| G_{2}$ by $u_{12}$ and $v_{12}$.

Obviously, the only vertices whose degrees are affected by the above operations are $u_{i}$ and $v_{i}$, for $i=1,2$. If we denote by $v_{12}$ the vertex of $G_{1} \cdot G_{2}$ obtained by identifying $v_{1}$ and $v_{2}$, we have the following expressions:

$$
\begin{aligned}
& d_{G_{1} \cdot G_{2}}\left(v_{12}\right)=d_{G_{1}}\left(v_{1}\right)+d_{G_{2}}\left(v_{2}\right) ; \\
& d_{G_{1} \sim G_{2}}\left(v_{1}\right)=d_{G_{1}}\left(v_{1}\right)+1 ; \\
& d_{G_{1} \sim G_{2}}\left(v_{2}\right)=d_{G_{2}}\left(v_{2}\right)+1 ; \\
& d_{G_{1} \| G_{2}}\left(u_{12}\right)=d_{G_{1}}\left(u_{1}\right)+d_{G_{2}}\left(u_{2}\right)-1 ; \\
& d_{G_{1} \| G_{2}}\left(v_{12}\right)=d_{G_{1}}\left(v_{1}\right)+d_{G_{2}}\left(v_{2}\right)-1 .
\end{aligned}
$$

The following results are direct consequences of the above observations.

\section{Proposition 1}

$$
\begin{aligned}
& N K\left(G_{1} \cdot G_{2}\right)=N K\left(G_{1}\right) N K\left(G_{2}\right) \frac{d_{G_{1}}\left(v_{1}\right)+d_{G_{2}}\left(v_{2}\right)}{d_{G_{1}}\left(v_{1}\right) d_{G_{2}}\left(v_{2}\right)} ; \\
& N K\left(G_{1} \sim G_{2}\right)=N K\left(G_{1}\right) N K\left(G_{2}\right) \frac{\left(d_{G_{1}}\left(v_{1}\right)+1\right)\left(d_{G_{2}}\left(v_{2}\right)+1\right)}{d_{G_{1}}\left(v_{1}\right) d_{G_{2}}\left(v_{2}\right)} ;
\end{aligned}
$$

$$
\begin{aligned}
& N K\left(G_{1} \| G_{2}\right)=N K\left(G_{1}\right) N K\left(G_{2}\right) \\
& \quad \frac{\left(d_{G_{1}}\left(u_{1}\right)+d_{G_{2}}\left(u_{2}\right)-1\right)\left(d_{G_{1}}\left(v_{1}\right)+d_{G_{2}}\left(v_{2}\right)-1\right)}{d_{G_{1}}\left(u_{1}\right) d_{G_{2}}\left(u_{2}\right) d_{G_{1}}\left(v_{1}\right) d_{G_{2}}\left(v_{2}\right)} .
\end{aligned}
$$

An alternative way of writing the second result is

$$
\begin{gathered}
N K\left(G_{1} \sim G_{2}\right)=N K\left(G_{1}\right) N K\left(G_{2}\right)+N K\left(G_{1} \cdot G_{2}\right)+ \\
N K^{\left(V\left(G_{1}\right)-v_{1}\right)}\left(G_{1}\right) N K^{\left(V\left(G_{2}\right)-v_{2}\right)}\left(G_{2}\right)
\end{gathered}
$$

The results for splice can be in a straightforward way generalized to more than two operands. If we have graphs $G_{1}, \ldots, G_{k}$ and $v_{i} \in V\left(G_{i}\right)$ for each $i=1, . ., k$, then their splice in vertices $v_{i}$ is obtained by identifying all $k$ vertices $v_{i}$.

Corollary 2

$$
N K\left(G_{1} \cdot G_{2} \cdot \ldots \cdot G_{k}\right)=\frac{\sum_{i=1}^{k} d_{G_{i}}\left(v_{i}\right)}{\prod_{i=1}^{k} d_{G_{i}}\left(v_{i}\right)} \prod_{i=1}^{k} N K\left(G_{i}\right) .
$$

If we have $k$ copies of the same graph $G$ and splice them at the same vertex $v$, we obtain $G^{k}$, the $k$-th splicepower of $G$. The above result then simplifies to

$$
N K\left(G^{k}\right)=\frac{k[N K(G)]^{k}}{d_{G}(v)^{k-1}} .
$$

By considering links of more than two graphs we arrive at the next class of composite graphs considered here, the chain (or bridge) graphs.

\section{Chains and Necklaces}

Let $G_{i}, 1 \leq i \leq k$, be some graphs and $v_{i} \in V\left(G_{i}\right)$. A chain graph denoted by $G=G\left(G_{1}, \ldots, G_{k}, v_{1}, \ldots, v_{k}\right)$ is obtained from the union of the graphs $G_{i}, i=1, \ldots, k$, by adding the edges $v_{i} v_{i+1} \quad 1 \leq i \leq k-1$, see Figure 1 . Then $|V(G)|=\sum_{i=1}^{k}\left|V\left(G_{i}\right)\right|$ and $|E(G)|=(k-1)+$ $\sum_{i=1}^{k}\left|E\left(G_{i}\right)\right|$. By adding the edge $v_{k} v_{1}$ to a chain graph we obtain the corresponding necklace $G_{0}=G_{0}\left(G_{1}, \ldots, G_{k}\right.$, $\left.v_{1}, \ldots, v_{k}\right)$.

One can see that $G\left(G_{1}, G_{2}, v_{1}, v_{2}\right) \cong\left(G_{1} \sim G_{2}\right)\left(v_{1}\right.$, $\left.v_{2}\right)$.

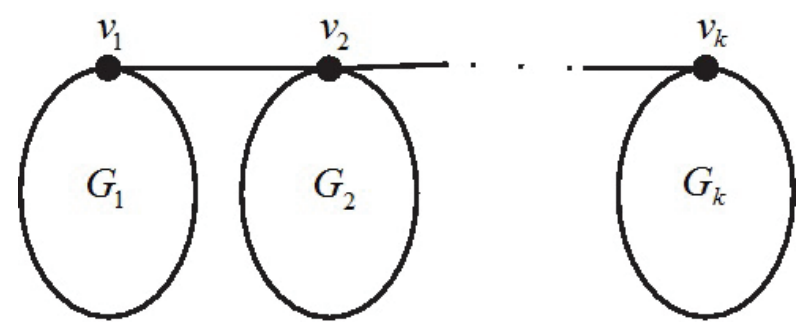

Figure 1. The chain graph $G=G\left(G_{1}, \ldots, G_{k}, v_{1}, \ldots, v_{k}\right)$. 
It is worth noting that the above specified classes of chain graphs and necklaces embrace, as special cases, all trees (among which are the molecular graphs of alkanes) and all unicyclic graphs (among which are the molecular graphs of monocycloalkanes). Also the molecular graphs of many polymers and dendrimers are chain graphs. Further, when all $G_{i}$ are equal to $G$ and all $v_{i}$ are equal, we have the rooted products of $P_{k}$ and $G$ and of $C_{k}$ and $G$.

It is clear that the root vertices are the only ones whose degrees are affected by the chain and necklace construction. Hence,

$$
\begin{aligned}
& d_{G}(u)= \begin{cases}d_{G_{i}}(u) & \text { if } u \in V\left(G_{i}\right) \text { and } u \neq v_{i} \\
d_{G_{i}}\left(v_{i}\right)+1 & \text { if } u=v_{i}, i=1, k \\
d_{G_{i}}\left(v_{i}\right)+2 & \text { if } u=v_{i}, 2 \leq i \leq k-1 .\end{cases} \\
& d_{G_{0}}(u)= \begin{cases}d_{G_{i}}(u) & \text { if } u \in V\left(G_{i}\right) \text { and } u \neq v_{i} \\
d_{G_{i}}\left(v_{i}\right)+2 & \text { if } u=v_{i}, 1 \leq i \leq k .\end{cases}
\end{aligned}
$$

Theorem 3

$$
\begin{gathered}
N K\left(G\left(G_{1}, \ldots, G_{k}, v_{1}, \ldots, v_{k}\right)\right)=\left(d_{G_{1}}\left(v_{1}\right)+1\right)\left(d_{G_{k}}\left(v_{k}\right)+1\right) \\
\cdot \prod_{i=2}^{k-1}\left(d_{G_{1}}\left(v_{i}\right)+2\right) \prod_{i=1}^{n} N K^{\left(V\left(G_{i}\right)-v_{i}\right)}\left(G_{i}\right) .
\end{gathered}
$$

Theorem 4

$$
\begin{gathered}
N K\left(G_{0}\left(G_{1}, \ldots, G_{k}, v_{1}, \ldots, v_{k}\right)\right)=\prod_{i=1}^{k}\left(d_{G_{1}}\left(v_{i}\right)+2\right) \\
\cdot \prod_{i=1}^{n} N K^{\left(V\left(G_{i}\right)-v_{i}\right)}\left(G_{i}\right) .
\end{gathered}
$$

In both cases the proof follows immediately by using the definition of the truncated $N K$ index, and we omit the details.

\section{Join}

The join (sometimes also called the sum) of two graphs $G_{1}$ and $G_{2}$ is obtained by taking their union and adding all possible edges between $V\left(G_{1}\right)$ and $V\left(G_{2}\right)$. We denote it by $G_{1} \nabla G_{2}$. When one of the graphs is $K_{1}$, the join of $K_{1}$ and $G$ is called the suspension of $G$. The degree of a vertex of $G$ in its suspension increases by one, while the degree of the vertex of $K_{1}$ is equal to $|V(G)|=n$. Hence the Narumi-Katayama index of $K_{1} \nabla G$ is given by

$$
N K\left(K_{1} \nabla G\right)=n \prod_{i=1}^{n}\left(d_{G}\left(v_{i}\right)+1\right) .
$$

The product on the right-hand side of the above formula can be expressed in terms of truncated NarumiKatayama indices with respects to all subsets of $V(G)$. The result follows by expanding the product into a sum of $2^{n}$ terms and noting that the products of degrees of each of $2^{n}$ subsets of $V(G)$ appear exactly once in the sum.

Proposition 5

$$
N K\left(K_{1} \nabla G\right)=n \sum_{U \subseteq V(G)} N K^{(U)}(G) .
$$

The above result can be straightforwardly generalized to the case when one of the components of a join is the set of $m$ independent vertices, i.e., the complement $\bar{K}_{m}$ of the complete graph $K_{m}$.

Proposition 6

$$
N K\left(\bar{K}_{m} \nabla G\right)=n^{m}\left(\sum_{U \subseteq V(G)} N K^{(U)}(G) m^{n-|U|}\right) .
$$

A closer look on the above formula should reveal that all effects of the independence of vertices of $\bar{K}_{m}$ are concentrated in the $n^{m}$ term. Hence, the contribution of vertices of one component in a join of two graphs depends only on the number of vertices in the other component, and not on its internal structure. From this observation we can deduce the formula for the general case.

\section{Proposition 7}

Let $G_{1}$ and $G_{2}$ be two graphs with $n_{1}$ and $n_{2}$ vertices, respectively. Then

$$
\begin{gathered}
N K\left(G_{1} \nabla G_{2}\right)=\left(\sum_{U_{1} \subseteq V\left(G_{1}\right)} N K^{\left(U_{1}\right)}\left(G_{1}\right) n_{2}^{n_{1}-\left|U_{1}\right|}\right) \\
\cdot\left(\sum_{U_{2} \subseteq V\left(G_{2}\right)} N K^{\left(U_{2}\right)}\left(G_{2}\right) n_{1}^{n_{2}-\left|U_{2}\right|}\right) .
\end{gathered}
$$

The results of this subsection could be further generalized to joins of more than two graphs, but we leave that to the interested reader. Instead, we use them to derive formulas for the Narumi-Katayama index of a corona of two graphs.

\section{Corona}

The corona of two graphs $G$ and $H$ is the graph obtained by taking $|V(G)|$ copies of $H$ and connecting each vertex in the $i$-th copy of $H$ to the vertex $v_{i}$ of $G$. It is usually denoted by $G \circ H$. (We have used $G$ and $H$ instead of $G_{1}$ and $G_{2}$ in order to stress the fact that the components enter their corona in an asymmetric way.) Hence, a 
corona is a collection of $n$ suspensions of $H$ on a scaffold provided by $G$. This is reflected in the formula for its Narumi-Katayama index.

\section{Proposition 8}

Let $G$ and $H$ be two graphs with $n$ and $m$ vertices, respectively. Then

$$
\begin{gathered}
N K(G \circ H)=\left(\sum_{U \subseteq V(G)} N K^{(U)}(G) m^{n-|U|}\right) \\
\cdot\left(\sum_{W \subseteq V(H)} N K^{(W)}(H)\right)^{n} .
\end{gathered}
$$

\section{Composition}

The composition of two graphs $G$ and $H$ is the graph with vertex set $V(G) \times V(H)$, and the vertex $u=\left(u_{1}, v_{1}\right)$ is adjacent to the vertex $v=\left(u_{2}, v_{2}\right)$ whenever either $u_{1} u_{2} \in$ $E(G)$ or $u_{1}=u_{2}$ and $v_{1} v_{2} \in E(H)$. This graph operation is denoted by $G[H]$. So the degree of the vertex $(u, v)$ in $G[H]$ is $d_{G[H]}(u, v)=d_{H}(v)+m d_{G}(u)$, where $m$ is the number of vertices of $H$. The composition of two graphs is also known as graph substitution, a name that bears witness to the fact that $G[H]$ can be obtained from $G$ by substituting a copy of $H$, labeled $H_{w}$, for every vertex $w$ in $V(G)$ and then joining all vertices of $H_{w}$ with all vertices of $H_{w^{\prime}}$ if and only if $w w^{\prime} \in E(G)$, and there are no edges between vertices in $H_{u}$ and $H_{u^{\prime}}$ otherwise. Now by the above approach, one can see the Narumi-Katayama index of the composition of two graphs as follows:

\section{Proposition 9}

Let $G$ and $H$ be two graphs with $n$ and $m$ vertices, respectively. Then

$$
N K(G[H])=\prod_{u \in V(G)} \sum_{U \subseteq V(H)} N K^{(U)}(H)\left(m d_{G}(u)\right)^{m-|U|} .
$$

\section{Cartesian Product}

The Cartesian product $G_{1} \square G_{2}$ of graphs $G_{1}$ and $G_{2}$ is a graph such that $V\left(G_{1} \square G_{2}\right)=V\left(G_{1}\right) \times V\left(G_{2}\right)$, and any two vertices $\left(u_{1}, v_{1}\right)$ and $\left(u_{2}, v_{2}\right)$ are adjacent in $G_{1} \square G_{2}$ if and only if either $\left(u_{1}=u_{2}\right.$ and $v_{1}$ is adjacent with $\left.v_{2}\right)$, or $\left(v_{1}=v_{2}\right.$ and $u_{1}$ is adjacent with $\left.u_{2}\right)$. It is easy to see that $d_{G_{1} \square G_{2}}(u, v)=d_{G_{1}}(u)+d_{G_{2}}(v)$. According to the previous subsections, we can write the Narumi-Katayama index of the Cartesian product of two graphs $G_{1}$ and $G_{2}$ with $n$ and $m$ vertices, respectively, by

$$
\prod_{u \in V\left(G_{1}\right)} \sum_{U \subseteq V\left(G_{2}\right)} N K^{(U)}\left(G_{2}\right)\left(d_{G_{1}}(u)\right)^{m-|U|}
$$

or

$$
\prod_{v \in V\left(G_{2}\right)} \sum_{W \subseteq V\left(G_{1}\right)} N K^{(W)}\left(G_{1}\right)\left(d_{G_{2}}(v)\right)^{n-|W|} .
$$

So to preserve the symmetric of the formula for the Narumi-Katayama index of Cartesian product of two graphs, we have the next proposition.

\section{Proposition 10}

Let $G_{1}$ and $G_{2}$ be two graphs with $n$ and $m$ vertices, respectively. Then

$$
\begin{gathered}
N K\left(G_{1} \square G_{2}\right)=\frac{1}{2} \prod_{u \in V\left(G_{1}\right)} \sum_{U \subseteq V\left(G_{2}\right)} N K^{(U)}\left(G_{2}\right)\left(d_{G_{1}}(u)\right)^{m-|U|}+ \\
\frac{1}{2} \prod_{v \in V\left(G_{2}\right)} \sum_{W \subseteq V\left(G_{1}\right)} N K^{(W)}\left(G_{1}\right)\left(d_{G_{2}}(v)\right)^{n-|W|} .
\end{gathered}
$$

\section{APPLICATIONS}

\section{Spiro and Polyphenyl Hexagonal Chains}

A (poly)spiro compound is a polycyclic organic compound whose rings are connected by one atom. The rings may be of various lengths. The connecting atom, most often a carbon, is also called the spiroatom. Their graphs appear in the mathematical literature as cactus graphs; if a polyspiro compound is unbranched, the corresponding graph is also known as cactus chain. ${ }^{3}$ If all cycles (rings) are of the same length, we say that the chain is uniform. An example of a uniform (hexagonal) spiro chain of length 6 is shown in Figure 2. Let a hexagonal chain of length $h$ be denoted by $H_{h}$. From the first claim of Proposition 1 we obtain the recurrence for $N K\left(H_{h}\right)$,

$$
N K\left(H_{h+1}\right)=N K\left(H_{h}\right) N K\left(H_{1}\right) .
$$

This, together with the obvious initial condition $N K\left(H_{1}\right)=2^{6}=64$, yields the following result.

\section{Corollary 11}

$$
N K\left(H_{h}\right)=64^{h} .
$$

The same reasoning remains valid also when the

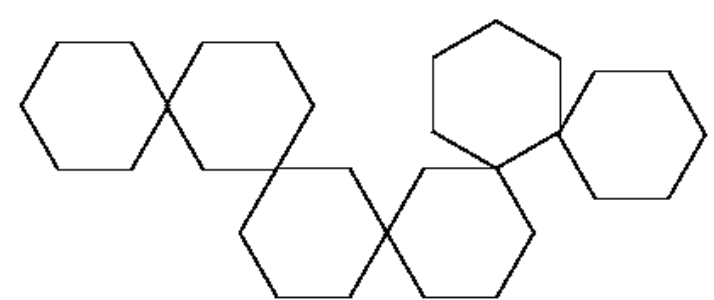

Figure 2. A hexagonal spiro chain of length 6. 


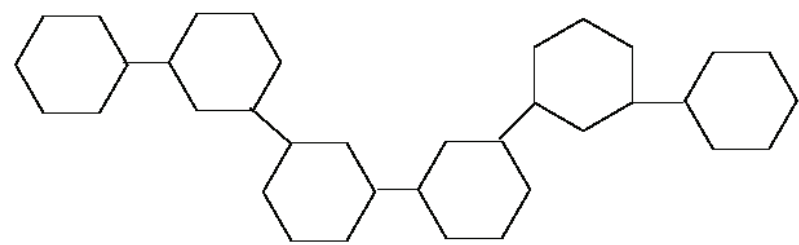

Figure 3. A polyphenyl hexagonal chain of length 6 .

cycles are not all of the same length. In general, if $G_{h}$ is a cactus graph with $h$ blocks in which every cut-vertex is shared by exactly two cycles, then

$$
N K\left(G_{h}\right)=2^{\left|V\left(G_{h}\right)\right|},
$$

regardless of the structure of $G_{h}$.

A class of polycyclic compounds in which two or more benzene rings are connected by a cut edge is known as polyphenyl compounds. Their graphs are called polyphenyl hexagonal chains. An example is shown in Figure 3. We denote such a chain with $h$ hexagons by $P P_{h}$. By using the second claim of Proposition 1 we immediately obtain

$$
N K\left(P P_{h}\right)=\left(\frac{9}{4}\right)^{h-1} N K\left(H_{h}\right),
$$

leading to the explicit expression $N K\left(P P_{h}\right)=(4 / 9) 144^{h}$.

\section{Catacondensed Benzenoids}

It is clear that the graph of any catacondensed benzenoid can be constructed by starting from a single hexagon and adding one hexagon at a time by the gate operation. If we denote a catacondensed benzenoid with $h$ hexagons by $B_{h}$, we obtain a recurrence for $N K\left(B_{h}\right)$ in the form

$$
N K\left(B_{h+1}\right)=\left(\frac{9}{16}\right) N K\left(B_{h}\right) N K\left(B_{1}\right)
$$

resulting in the explicit formula $N K\left(B_{h}\right)=$ $(9 / 16)^{h-1} N K\left(B_{1}\right)^{h}=(16 / 9) 36^{h}$. (See also Ref. 12.)

\section{Phenylenes and Their Hexagonal Squeezes}

The last class of object we consider here are phenylenes and their hexagonal squeezes as defined in Ref. 12. It is obvious that any phenylene can be constructed starting from a single hexagon and adding one hexagon at a time by iterating the two-step construction shown in Figure 4. Hence, we first link a hexagon to the already constructed graph, and then add an edge. We will need the following lemma that describes the effect of adding an edge to $G$.

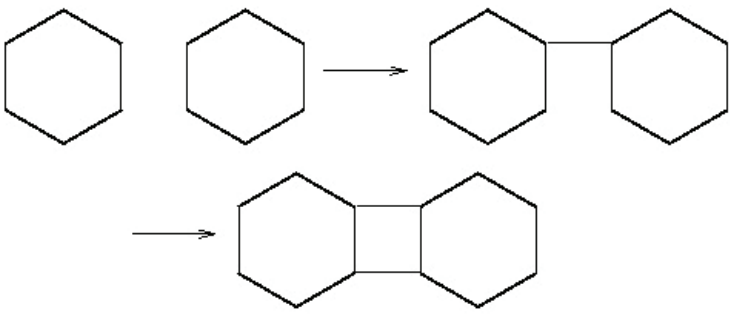

Figure 4. Two-step construction of a phenylene.

\section{Lemma 12}

Let $G$ be a connected graph and $u, v \in V(G)$ two nonadjacent vertices of $G$. Then

$$
N K(G+u v)=N K(G) \frac{(d(u)+1)(d(v)+1)}{d(u) d(v)} .
$$

By combining Lemma 12 with the second claim of Proposition 1 we obtain a recurrence for the NarumiKatayama indices of phenylenes and their hexagonal squeezes. It leads to explicit formulas that confirm the relationships between them established in Ref. 12 .

\section{Graph Irregularity}

Regularity of a graph is a binary property - a graph is either regular or not. However, while all regular graphs (of a given order and size) are equally regular, the nonregular graphs of the same order and size are not all equally far from being regular. There are several proposed measures of non-regularity of a graph. Most of them are based on measuring local discrepancies, i.e., quotients or differences of degrees of adjacent vertices. An example is the invariant, first introduced and studied by Albertson ${ }^{1}$ and called irregularity. The irregularity of a graph $G$ is defined as

$$
\operatorname{irr}(G)=\sum_{u v \in E(G)}\left|d_{G}(u)-d_{G}(v)\right|
$$

(The same quantity is sometimes called also the third Zagreb index.) Further examples are the arithmeticgeometric index and other indices based on combinations of various means of degrees of adjacent vertices.

Let $\mathrm{G}$ be a graph on $n$ vertices and $m$ edges. It is clear from the Arithmetic-Geometric Mean inequality that the Narumi-Katayama index of a graph cannot exceed the $n$-th power of the average degree of the graph $(2 m / n)^{n}$. Furthermore, the bound is attained if and only if $\mathrm{G}$ is regular. Hence, it is to be expected that a greater variability of degrees of vertices of $G$ will be reflected in a smaller value of its Narumi-Katayama 

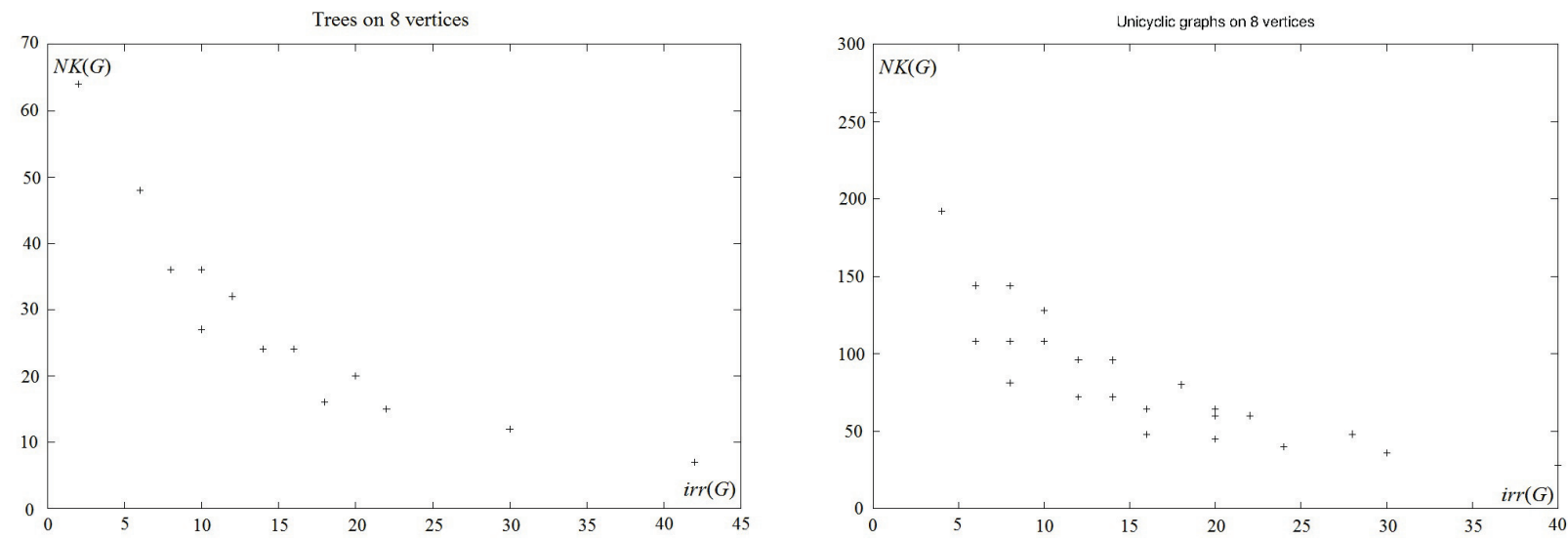

Figure 5. The Narumi-Katayama index $v s$. irregularity for trees (left) and unicyclic graphs (right) on 8 vertices.

index. This indeed seems to be the case. In Figure 5 we show the scatter-plot of $\operatorname{irr}(G) v s . N K(G)$ for all trees (left) and all unicyclic graphs (right) on 8 vertices. Although the exact nature of the relationship remains unexplored and the trend becomes less pronounced for denser graphs, it is clear that the Narumi-Katayama index can serve as a useful global measure of the graph irregularity.

\section{CONCLUSION}

The Narumi-Katayama index of a graph is defined as the product of degrees of all vertices. We studied how this quantity behaves under binary operations affecting vertex degrees. As our main result, we have obtained explicit formulas for values of the Narumi-Katayama index for several classes of graphs that arise from simpler graphs via some binary operations. The obtained results were then applied to several families of graphs that serve as models of unbranched hydrocarbon polymers. We have also investigated the potential of the Narumi-Katayama index as a measure of irregularity of graphs. We found that it performs well on trees and unicyclic graphs on a given number of vertices.

Acknowledgements. The authors would like to thank the referees for their valuable comments. Partial support by the Center of Excellence of Algebraic Hyperstructures and its Applications of Tarbiat Modares University (CEAHA) is gratefully acknowledged by A. Iranmanesh. Partial support of the
Ministry of Science, Education and Sport of the Republic of Croatia (Grants No. 177-0000000-0884 and 037-00000002779 ) is gratefully acknowledged by T. Došlić.

\section{REFERENCES}

1. M. O. Albertson, Ars Combin. 46 (1997) 219-225.

2. T. Došlić, Graph Theory Notes New York 48 (2005) 47-55.

3. T. Došlić and F. Måløy, Discrete Math. 310 (2010) 1676-1690.

4. I. Gutman, MATCH Commun. Math. Comput. Chem. 25 (1990) 131-140.

5. I. Gutman and M. Ghorbani, Appl. Math. Lett. 25 (2012) 1435-1438.

6. I. Gutman and H. Narumi, Coll. Sci. Pap. Fac. Sci. Kragujevac 11 (1990) 19-22.

7. W. Imrich and S. Klavžar, Product Graphs: Structure and Recognition, Wiley, New York, 2000.

8. D. J. Klein and V. R. Rosenfeld, MATCH Commun. Math. Comput. Chem. 64 (2010) 607-618.

9. D. J. Klein and V. R. Rosenfeld, The Narumi-Katayama degreeproduct index and the degree-product polynomial, in: I. Gutman and B. Furtula (Eds.), Novel Molecular Structure Descriptors Theory and Applications II, University of Kragujevac, Kragujevac, 2010, 79-90.

10. H. Narumi and M. Katayama, Memoirs Faculty Engin. Hokkaido Univ. 16 (1984) 209-214.

11. R. Todeschini and V. Consonni, Handbook of Molecular Descriptors, Wiley-VCH, Weinheim, 2000.

12. Ž. Tomović and I. Gutman, J. Serb. Chem. Soc. 66 (2001) 243-247.

13. N. Trinajstić, Chemical Graph Theory, CRC Press, Boca Raton, FL, 1992. 